
$\$$ Research Square
Preprints are preliminary reports that have not undergone peer review.
They should not be considered conclusive, used to inform clinical practice, or referenced by the media as validated information.

\title{
Arbuscular Mycorrhizal Fungi Regulate MAPK Pathway Genes Expression and Enhance Drought Tolerance of Populus Simonii $\times$ P. Nigra Seedlings
}

Jing Tao

Northwest A\&F University

Fengxin Dong

Northwest A\&F University

Yihan Wang

Northwest A\&F University

Hui Chen

South China Agricultural University

Ming Tang ( $\sim$ tangmingyl@163.com )

Northwest A\&F University

\section{Research Article}

Keywords: Poplar, Arbuscular mycorrhizal fungi, MAPK Drought

Posted Date: December 10th, 2021

DOI: https://doi.org/10.21203/rs.3.rs-1136524/v1

License: @ (i) This work is licensed under a Creative Commons Attribution 4.0 International License. Read Full License 


\section{Abstract}

Background: Arbuscular mycorrhizal fungi (AMF) form a symbiotic relationship with host plants, which can promote plants to absorb more water and nutrients, and thus improve the stress resistance of plants. Our study aimed to investigate the effects of Rhizophagus irregularis on Populus simonii $\times$ P. nigra seedlings under drought stress.

Results: The experiment was a completely random design with two water conditions (well-watered or drought stress) and two AMF treatments (inoculated with or without $R$. irregularis). Our results showed that mycorrhizal seedlings performed less oxidative damage and stronger tolerance of drought, which recorded higher photosynthesis and less concentrations of Malondialdehyde (MDA), $\mathrm{H}_{2} \mathrm{O}_{2}$, and proline under drought stress versus non-mycorrhizal seedlings. Under drought stress, AMF inoculation reduced soluble sugar concentration in leaves but promoted its accumulation in roots. The superoxide dismutase (SOD) activity in leaves and roots, and catalase (CAT) activity in roots of mycorrhizal seedlings were lower than nonmycorrhizal seedlings, but CAT activity in leaves of mycorrhizal seedlings was higher than non-mycorrhizal seedlings under drought stress. Drought stress and AMF inoculation both induced the expressions of MAPKs of $P$. simonii $\times$ P. nigra, but the expression patterns of $M A P K s$ under four treatments were obviously different.

Conclusions: Overall, our results demonstrated that mycorrhizal seedlings had less oxidative damage and stronger tolerance to drought. MAPKs expressions of P. simoniixP. nigra (PsnMAPKs) were induced by drought stress and AMF inoculation, and the expression patterns of PsnMAPKs in response to drought stress were different between mycorrhizal and non-mycorrhizal seedlings. Non-mycorrhizal seedlings may be adapted to drought by up-regulating MAPKs expressions leading to stomatal closure. Drought stress decreased serval PsnMAPKs expressions induced by AMF inoculation, which may be associated with mycorrhizal colonization.

\section{Introduction}

Plants in nature are constantly exposed to a variety of biotic and abiotic stresses. Among these abiotic stresses, drought stress is one of the most unfavorable factors for plants. Drought stress leads to the degradation of photosynthesis and the interruption of electron transport in thylakoids, and the closure of stomata leads to reduce carbon dioxide absorption and carbon flux [1], resulting in a decline in plant growth and productivity [2]. Meanwhile, drought stress causes a burst of reactive oxygen species (ROS) accumulation, disrupting cell metabolism [3]. Malondialdehyde (MDA) is an indicator to assess the level of lipid peroxidation in cell membranes. As one of the ROS, the decrease of $\mathrm{H}_{2} \mathrm{O}_{2}$ degradation ability in leaves may be the root cause of oxidative damage and membrane lipid peroxidation in plants under drought stress [4]. Under adversity conditions, proline will accumulate in plants and plays an important role in the defense mechanism of plants [5]. Soluble sugar is of great help to osmotic regulation, and also acts as a source of carbon [6]. In addition, plants also turn on their antioxidant enzyme system to clear ROS under drought stress [7]. Moreover, some signal transduction molecules such as $\mathrm{H}_{2} \mathrm{O}_{2}$, can active signal pathways during plants acclimation to drought [4].

Arbuscular Mycorrhiza (AM) is the most common symbiotic structure between plants and soil microorganisms. Arbuscular Mycorrhizal Fungi (AMF) exist in most natural habitats, and they provide a series 
of important ecological functions, especially to improve plant nutrition, production, stress resistance, soil fertility and the restoration of degraded lands [8-11]. The poplar (Populus spp.) has important economic value, which plays an increasingly important role in the greening of the country, the construction of farmland shelterbelts, and the construction of industrial timber forests, especially in the context of global atmospheric changes, the carbon sequestration benefits of poplars increasingly becoming the focus of attention [12].

As a main signal transducer, the mitogen-activated protein kinase (MAPK) cascade plays a vital role in drought stress [13]. In rice, the transcripts of OSMPK8, OSMPK7, OSMPK5, and OSMPK4 accumulated under drought stress $[14,15]$. It has been reported that GhMPK2 enhances the drought resistance of cotton through stomatal closure, which mainly responses to drought stress by reducing water loss and regulating intracellular osmotic pressure [16]. It is documented that OSDSM1 in rice may respond to drought stress mainly by regulating the ability of ROS scavenging [17]. Overexpression of GhMPK3 mainly enhances the drought tolerance of plants by regulating the activity of antioxidant enzymes [18]. Hence, the expressions of MAPKs are associated with drought tolerance of plants.

In arid and semi-arid areas, the water shortage has always been a key factor restricting agricultural and forestry production [19]. Therefore, improving drought resistance of woody plants is related to the forestry development in arid and semi-arid areas. In the past, the research on mycorrhiza to improve the drought tolerance of poplars mostly focused on root morphology, nutrient absorption, antioxidant system and photosynthetic system $[20,21]$. As far as we know, there is no report of mycorrhiza-induced MAPK genes of poplars. Our study aimed to investigate the effects of Rhizophagus irregularis on drought tolerance and MAPKs expressions of Populus simonii $\times$ P. nigra seedlings.

\section{Results}

\section{Effect of Drought and AMF inoculation on plant growth}

Mycorrhizal colonization was not found in the roots of uninoculated seedlings. The mycorrhizal colonization rate of the AMF-inoculated seedlings was $81.56 \% \pm 0.10 \%$ under WW treatment and $64.84 \% \pm 0.12 \%$ under DS treatment, respectively. DS treatment significantly inhibited the growth of seedlings, but the AMF inoculation promoted plant growth, which was reflected in the height of the plant increased by $79.11 \%$. In the case of WW treatment, AMF inoculation also increased the height of plants by $25.43 \%$ (Table 1). Under WW treatment, AMF inoculation significantly promoted the fresh weight of shoots, fresh weight of roots and total biomass, which increased by $45.59 \%, 29.96 \%$, and $39.97 \%$, respectively (Table 1). Under DS treatment, AMF inoculation dramatically increased fresh weight of roots by $99.56 \%$ but had no significant effect on fresh weight of shoots and total biomass. Notably, DS treatment increased the root-shoot ratio, but inoculation with AMF reduced the root-shoot ratio by $24.00 \%$.

Table 1. Effects of drought and $R$. intraradices on growth of $P$. simoniixP. nigra seedlings 


\begin{tabular}{|lllllll|}
\hline Treatments & $\begin{array}{l}\text { AM } \\
\text { colonization } \\
\text { rate }(\%)\end{array}$ & $\begin{array}{l}\text { Plant } \\
\text { height }(\mathrm{cm})\end{array}$ & $\begin{array}{l}\text { Fresh } \\
\text { Weight of } \\
\text { Shoot }(\mathrm{g})\end{array}$ & $\begin{array}{l}\text { Fresh } \\
\text { Weight of } \\
\text { Root }(\mathrm{g})\end{array}$ & Root/Shoot & $\begin{array}{l}\text { Total } \\
\text { Biomass (g) }\end{array}$ \\
\hline WW-NM & - & $33.23 \pm 2.06 \mathrm{~b}$ & $21.33 \pm 2.90 \mathrm{~b}$ & $11.95 \pm 1.20 \mathrm{~b}$ & $0.57 \pm 0.06 \mathrm{c}$ & $33.28 \pm 3.79 \mathrm{~b}$ \\
\hline WW-AM & $81.56 \pm 0.10 \mathrm{a}$ & $41.68 \pm 0.62 \mathrm{a}$ & $31.06 \pm 3.35 \mathrm{a}$ & $15.53 \pm 2.09 \mathrm{a}$ & $0.50 \pm 0.02 \mathrm{c}$ & $46.58 \pm 5.38 \mathrm{a}$ \\
\hline DS-NM & - & $3.95 \pm 0.42 \mathrm{~d}$ & $1.47 \pm 0.3 \mathrm{c}$ & $2.86 \pm 0.21 \mathrm{~d}$ & $2.00 \pm 0.32 \mathrm{a}$ & $4.33 \pm 0.48 \mathrm{c}$ \\
\hline DS-AM & $64.84 \pm 0.12 \mathrm{~b}$ & $7.08 \pm 0.39 \mathrm{c}$ & $3.78 \pm 0.35 \mathrm{c}$ & $5.71 \pm 0.29 \mathrm{c}$ & $1.52 \pm 0.06 \mathrm{~b}$ & $9.49 \pm 0.64 \mathrm{c}$ \\
\hline
\end{tabular}

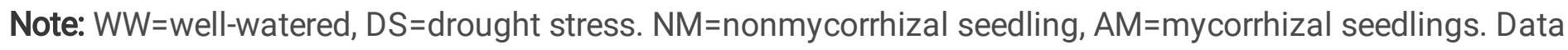
(means $\pm \mathrm{SD}, \mathrm{n}=4$ ) followed by different letters in the column indicate significant differences $(P<0.05)$ between treatments.

\section{Effect of Drought and AMF inoculation on Photosynthesis}

Compared with WW treatment, drought stress significantly decreased Pn, gs and Tr of seedlings. AMFinoculation significantly enhanced $\mathrm{Pn}$, gs, and $\operatorname{Tr}$ by $34.66 \%, 78.36 \%$, and $47.36 \%$, respectively, under wellwatered conditions. Under drought conditions, AMF inoculation also significantly enhanced Pn, gs, and Tr by $137.79 \%, 195.81 \%$, and $278.75 \%$, respectively, compared with NM seedlings. However》there was no significant difference in the $\mathrm{Ci}$ among the four treatments (Table 2).

Table 2. Effects of drought and $R$. irregularis on photosynthetic parameters of $P$. simoniixP. nigra seedlings.

\begin{tabular}{|lllll|}
\hline Treatments & $\begin{array}{l}\text { Pn }\left(\mu \mathrm{mol} / \mathrm{m}^{2} \text { per }\right. \\
\text { second })\end{array}$ & $\begin{array}{l}\text { gs }\left(\mu \mathrm{mol} / \mathrm{m}^{2} \text { per }\right. \\
\text { second })\end{array}$ & $\mathrm{Ci}(\mu \mathrm{mol} / \mathrm{mol})$ & $\begin{array}{l}\operatorname{Tr}\left(\mathrm{mmol} / \mathrm{m}^{2} \text { per }\right. \\
\text { second })\end{array}$ \\
\hline WW-NM & $8.35 \pm 0.79 \mathrm{~b}$ & $70 \pm 0.01 \mathrm{~b}$ & $241.56 \pm 10.84 \mathrm{a}$ & $1.70 \pm 0.11 \mathrm{~b}$ \\
\hline WW-AM & $11.25 \pm 1.95 \mathrm{a}$ & $120 \pm 0.04 \mathrm{a}$ & $250.57 \pm 29.10 \mathrm{a}$ & $2.51 \pm 0.74 \mathrm{a}$ \\
\hline DS-NM & $2.95 \pm 0.47 \mathrm{c}$ & $20 \pm 0.00 \mathrm{c}$ & $219.77 \pm 26.33 \mathrm{a}$ & $0.65 \pm 0.07 \mathrm{c}$ \\
\hline DS-AM & $7.02 \pm 0.98 \mathrm{~b}$ & $60 \pm 0.00 \mathrm{~b}$ & $225.57 \pm 15.98 \mathrm{a}$ & $1.56 \pm 0.28 \mathrm{~b}$ \\
\hline
\end{tabular}

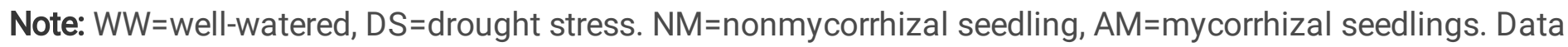
(means $\pm S D, n=4)$ followed by different letters in the column indicate significant differences $(P<0.05)$ between treatments.

\section{Effect of Drought and AMF inoculation on MDA and $\mathrm{H}_{2} \mathrm{O}_{2}$ concentrations}

Compared with WW treatment, the MDA concentrations in leaves and roots were found to be significantly increased under DS treatment. Under WW treatment, no matter whether it was inoculated or not, there was no significant difference in MDA concentration in leaves and roots. Whereas under DS treatment, AMF inoculation obviously decreased MDA concentration by $23.56 \%$ in leaves, and $47.2 \%$ in roots (Figure 1 ). Under the two water treatments, there was no significant difference in the concentration of $\mathrm{H}_{2} \mathrm{O}_{2}$ in the leaves of $A M$ and $N M$ seedlings, but the concentration of $\mathrm{H}_{2} \mathrm{O}_{2}$ in the leaves of $\mathrm{NM}$ seedlings was dramatically increased by $32.97 \%$ 
under DS treatment, while the concentration of $\mathrm{H}_{2} \mathrm{O}_{2}$ in the leaves of AM seedlings was also increased by DS treatment, but it did not reach a significant level. Under WW treatment, there was no significant difference in the concentration of $\mathrm{H}_{2} \mathrm{O}_{2}$ in the roots of the $\mathrm{NM}$ and $\mathrm{AM}$ seedlings. However, under DS treatment, the concentration of $\mathrm{H}_{2} \mathrm{O}_{2}$ in the roots of $\mathrm{NM}$ seedlings was dramatically increased by $45.41 \%$. Although DS treatment also increased the concentration of $\mathrm{H}_{2} \mathrm{O}_{2}$ in the roots of $\mathrm{AM}$ seedlings, it did not reach a significant level (Figure 2).

\section{Effect of Drought and AMF inoculation on Osmotic adjustment substances}

Compared with WW treatment, the proline concentrations in leaves and roots were found to be enhanced obviously under DS treatment. Under WW treatment, AMF inoculation had no significant effect on proline concentration. Whereas under DS treatment, AMF inoculation obviously decreased proline concentration by $27.42 \%$ in leaves, and $52.24 \%$ in roots (Figure 3 ). Under WW treatment, AMF inoculation had no significant effect on soluble sugar concentration. It is worth noting that AMF inoculation dramatically reduced soluble sugar concentration in leaves by $20.75 \%$ under DS treatment, compared with NM seedlings. Contrary to the result in the leaves, AMF inoculation dramatically enhanced soluble sugar concentration by $129.28 \%$ under DS treatment (Figure 4).

\section{Effect of Drought and AMF inoculation on Antioxidant Enzymes Activities}

DS treatment significantly enhanced SOD activity in leaves, but SOD activity in leaves of AM seedlings was lower versus NM seedlings under both water conditions. Under WW treatment, there was no significant difference in SOD activity in leaves between NM and AM seedlings. DS treatment enhanced SOD activity in roots, but SOD activity of AM seedlings was also lower versus NM seedlings (Figure 5A). Under WW treatment, there was no significant difference in CAT activity in leaves between NM and AM seedlings. DS treatment significantly enhanced CAT activity in leaves of AM seedlings but had no significant effect on NM seedlings. Notably, CAT activity in roots of AM seedlings was dramatically higher than NM seedlings under WW treatment. DS treatment significantly enhanced CAT activity in roots of NM seedlings but had no significant effect on AM seedlings (Figure 5B).

\section{Effect of Drought and AMF inoculation on expressions of MAPK genes}

According to the homology with MAPKs of $A$. thaliana and rice, the MAPKs of $P$. simoniixP. nigra (PsnMAPKs) were divided into four groups: A, B, C, and D (Figure 6). Phylogenetic analysis showed that PsnMAPK20-1 belonged to group A, PsnMAPK7-1 and PsnMAPK7-2 belonged to group C, and other PsnMAPKs were attributed to group $\mathrm{D}$, without genes belonged to group $\mathrm{B}$. The notable PsnMAPKs in leaves were PsnMAPK7-1 and PsnMAPK20-1, belonging to group $\mathrm{C}$ and $\mathrm{A}$. The effects of DS treatment on the expressions of them were significantly different between NM and AM seedlings. The expressions of these two genes dramatically increased in NM seedling leaves under DS treatment, but PsnMAPK7-1 was down-regulated in AM seedling leaves. Moreover, DS treatment did not affect the expression of PsnMAPK20-1 in AM seedling leaves to a significant level. The PsnMAPKs of group D in NM and AM seedling leaves were down regulated by DS treatment. In addition, the effects of inoculation on the expression of PsnMAPK7-1 in leaves were also significantly different between both water treatment. Under WW treatment, AMF inoculation increased the 
expression of PSnMAPK7-1 in leaves, but under DS treatment, AMF inoculation decreased the expression of PSnMAPK7-1. However, the expression of PSnMAPK20-1 in leaves was down-regulated by AMF inoculation under both water treatments. Additionally, the expressions of several PsnMAPKs in group D increased dramatically after AMF inoculation under WW treatment, including PSnMAPK19-1, PSnMAPK20-2, PsnMAPK12, PsnMAPK17(Figure 7A).

In the present study, we observed that more PsnMAPKs were affected by DS treatment in roots versus leaves. DS treatment increased the expressions of all PSnMAPKs in roots of NM seedlings except PSnMAPK15-2 and PsnMAPK20-1. Among these MAPKs, PsnMAPK7-1 and PSnMAPK7-2 in group C performed different responses to drought between NM and AM roots. DS treatment increased the expressions of PSnMAPK7-1 and PsnMAPK7-2 in NM roots but decreased them in AM roots. The expressions of PsnMAPK15-2, PsnMAPK7-1, PSnMAPK3-2, PSnMAPK12 and PSnMAPK17 were dramatically increased by AMF inoculation under WW treatment. However, under DS treatment, except PsnMAPK15-2 and PsnMAPK3-2, the expressions of other $M A P K s$ were decreased by AMF inoculation. Interestingly, the effect of DS treatment on the expressions of PSnMAPK15-2 were obviously different between NM and AM roots. DS treatment dramatically reduced the expression of PSnMAPK15-2 in AM roots but did not significantly affect the expression in NM roots (Figure 7B).

\section{Discussion}

\section{Plant growth}

Our study confirmed that soil drought decreases the mycorrhizal colonization rate, it may be because longterm drought stress inhibits mycorrhiza development [22]. Earlier studies suggested that drought stress strongly inhibits plant growth, while AM symbiosis considerably relieves the negative effects on plant growth [23]. In line with previous studies, our results showed that AM seedlings had a higher height than NM seedlings under two water conditions. Moreover, AMF inoculation under drought stress had a greater promotion on growth than well-watered conditions. Under well-watered conditions, AMF inoculation enhanced the fresh weight of shoots and roots and total biomass. In the present study, only the fresh weight of roots of AM seedlings was obviously higher versus NM seedlings. Drought stress causes changes in biomass allocation between shoots and roots of plants, and promotes photosynthates transfer to roots, resulting in an increase in root-shoot ratio [24], as shown in our study, root-shoot ratio significantly increased under drought conditions. Meanwhile, we noticed that root-shoot ratio of NM seedlings was higher than AM seedlings, which is consistent with the previous study [25]. In short, our results demonstrated that AMF has a positive effect on plant growth, especially on roots and under drought conditions.

\section{Photosynthesis}

Previous studies have reported that drought stress can reduce the Pn, gs, $\operatorname{Tr}[26,27]$. Plant growth and carbohydrate accumulation depend on photosynthesis. A lot of studies have shown that the Pn of mycorrhizal plants is higher than that of non-mycorrhizal plants, and they also have higher gs [28]. In response to drought stress, stomatal closure is an rapid response to avoid water loss, although it leads to negative effects on photosynthesis [29]. Stomata regulate carbon assimilation rate and water loss, but drought stress reduces 
gs [30]. AM symbiosis can change the stomatal conductance of plants, which is a reason for the enhance of Pn. Our findings are directly in line with previous findings, we observed that AM seedlings had higher Pn, gs, and Tr versus NM seedlings under two water conditions. AMF inoculation improved gas exchange by increasing the stomatal conductance and transpiration in leaves, which may be related to the increase in nutrient and water uptake caused by AMF [31]. Meanwhile, we found that there was no significant difference in $\mathrm{Ci}$, which is in accord with Chen et al. [32].

\section{Oxidative damage}

In previous studies, Pelargonium gravolens seedlings were inoculated with AMF under drought stress and then the accumulation of MDA was dramatically reduced [33]. The research on wheat also showed that AMF inoculation significantly reduced the MDA concentration under drought stress [34]. A same result was reached by our study, suggesting that oxidative damage of AM seedlings was slighter versus NM seedlings.

Furthermore, this effect was more obvious in roots than leaves. As one of the ROS, drought stress dramatically increased the accumulation of $\mathrm{H}_{2} \mathrm{O}_{2}$ in the leaves and roots of $\mathrm{NM}$ seedlings, but its effect on $\mathrm{H}_{2} \mathrm{O}_{2}$ homeostasis of AM seedlings did not reach a significant level. Moreover, AM roots recorded a lower level of $\mathrm{H}_{2} \mathrm{O}_{2}$ under drought stress. Overall, our findings are in accordance with findings reported by Mo et al. [35] and Rani et al. [34].

\section{Osmotic adjustment substances}

In the existing studies, the results about the changes of proline of mycorrhizal plants under drought stress are inconsistent. Studies by Zhang et al. [36] on Eriobotrya japonica and Tyagi et al. [37] on Eleusine coracana showed that the roots of AM plants accumulated more proline versus non-mycorrhizal plants under drought stress, and lipid peroxides of mycorrhizal plants were less than non-mycorrhizal plants under drought stress. Whereas, there are also some opposite results, in Erythrina variegata [38], Knautia arvensis [39], Pistacia vera [40], and Poncirus trifoliata [41], after inoculation with AMF, the accumulation of proline was lower than nonmycorrhizal plants. A similar pattern of results was obtained in our study, AM seedlings recorded a lower proline concentration than NM seedlings under drought stress. This possibly because AM seedlings have better water status and growth than NM seedlings under drought conditions, so they are less stressed.

Yooyongwech et al. [42] found that the soluble sugar concentration in mycorrhizal Ipomoea batatas was increased, and the accumulation in roots was higher than leaves, indicating that there is a negative correlation between osmotic potential and soluble sugar accumulation in plants. In the present study, under well-watered conditions, AMF inoculation had no significant effect on soluble sugar concentration. A novel finding is that the accumulation of soluble sugar in the leaves and roots of AM seedlings showed different patterns. The concentration of soluble sugar in leaves of AM seedlings was lower than NM seedlings, but the accumulation in the AM roots was higher than NM roots. The accumulation of sugar in mycorrhizal plants is caused by the enhancement of photosynthesis capacity under osmotic stress [43]. AMF inoculation can promote the transportation of carbohydrate from leaves to roots under drought conditions. Meanwhile, drought stress can also limit sugar transport from the roots to extraradical hyphae, which leads to accumulation increased in AM roots.

\section{Antioxidant enzyme activity}


Plants have a physiological mechanism to respond to oxidative damage by inducing the production of antioxidant enzymes [7]. When plants exposed to drought stress, they mobilize antioxidant systems to remove ROS. The SOD activity in leaves was dramatically increased by drought stress. Under drought conditions, SOD activity in roots of AM seedlings was lower versus NM seedlings, which is inconsistent with the previous results of Zou et al. [44] and Gong et al. [45]. This may be related to a lower oxidative burst in AM seedlings, which is supported by the result of the accumulation of MDA. The CAT activity is positively correlated with the ability of plants to remove $\mathrm{H}_{2} \mathrm{O}_{2}$. Under two water conditions, CAT activity in AM leaves was higher versus NM leaves, indicating that AMF inoculation enhanced the ability of $\mathrm{H}_{2} \mathrm{O}_{2}$ removal in leaves. Overall, our findings are in accordance with findings reported by Rani et al. [46], Zou et al. [44], and Gong et al. [45]. Drought stress leads to accumulation of $\mathrm{H}_{2} \mathrm{O}_{2}$, which is cleared rely on CAT. CAT activity in the roots of NM seedlings was higher versus AM roots under drought stress, possibly because less $\mathrm{H}_{2} \mathrm{O}_{2}$ was accumulated in AM roots.

\section{Quantitative expressions of PsnMAPKs}

Previous studies have showed that MAPK participate in response to drought stress [47, 48]. We determined that drought stress can affect the expressions of MAPKs of $P$. simonii $\times P$. nigra. In the present study, drought stress induced the expressions of PSnMAPK7-1 and PSnMAPK20-1 in leaves of NM seedlings, implying they are drought response genes in leaves. OSMAPK5 homologous to PSnMAPK20-1 has been confirmed to be activated by drought stress [14]. Hence, plants may adapt to drought stress by activating MAPK genes. Interestingly, under drought stress, the expressions of PSnMAPK7-1 and PsnMAPK20-1 were down-regulated by AMF inoculation exactly. It has been reported that GhMPK2 regulates drought stress via stomatal closure [49]. As shown in our study, NM seedlings recorded a lower gs under drought conditions, as compared with AM seedlings. In addition, we observed that AMF inoculation activated several MAPKs unrelated to drought in leaves, which is an interesting phenomenon. Previous studies only analyzed on MAPKs in roots affected by AMF inoculation [50,51], further work should also analyze those MAPKs in leaves up-regulated by AMF inoculation.

Drought stress induced more MAPKs in roots than leaves, we observed that drought stress increased dramatically the expressions of all MAPKs in roots of NM seedlings except PsnMAPK15-2 and PsnMAPK20-1, indicating the roots are stressed seriously. However, our study showed that the expressions of PsnMAPKs in roots were down-regulated by AMF inoculation under drought stress, except that PsnMAPK15-2 and PsnMAPK3-2 did not reach significant levels. ROS, a signaling molecule for response to stress, is an activator of MAPK, and previous studies have demonstrated that $\mathrm{H}_{2} \mathrm{O}_{2}$ can activate MAPK $[47,52,53]$. Moreover, MAPK overexpression can promote the activation of antioxidant defense system, thus reducing $\mathrm{H}_{2} \mathrm{O}_{2}$ level in plants [54]. The present results also suggested that NM roots recorded a higher level $\mathrm{H}_{2} \mathrm{O}_{2}$ and higher activity of antioxidant enzyme versus AM roots under drought stress. Our study confirmed that AMF inoculation affected the expressions of PsnMAPKs in roots, analogous results have been reported by Liu et al. [50] on Glycine max and Huang et al. [51] on Malus hupehensis. In our results, AMF inoculation induced the expressions of PsnMAPK15-2, PSnMAPK7-1, PsnMAPK3-2, PSnMAPK12 and PSnMAPK17 in roots under wellwatered conditions. Furthermore, drought stress decreased the expressions of PSnMAPK15-2 and PsnMAPK71 in AM roots, implying they may be associated with mycorrhization, which is inhibited by drought stress, but this should be demonstrated in further work. 


\section{Conclusion}

Overall, our results demonstrated an effective help of AMF on P. simoniixP. nigra seedlings under drought stress, reflecting in improvement of photosynthesis and a lower level of MDA and $\mathrm{H}_{2} \mathrm{O}_{2}$, which suggested mycorrhizal seedlings had less oxidative damage and stronger tolerance to drought. AMF inoculation promoted soluble sugar accumulated in roots under drought stress. In the present study, we confirmed that $M A P K s$ expressions of $P$. simoniixP. nigra were induced by drought stress and AMF inoculation, and the expression patterns of PSnMAPKs in response to drought stress were different between mycorrhizal and nonmycorrhizal seedlings. Non-mycorrhizal seedlings may be adapted to drought by up-regulating PSnMAPKs expressions leading to stomatal closure. Drought stress decreased serval PsnMAPKs expressions induced by AMF inoculation, which may be associated with mycorrhizal colonization. Furthermore, it is a question of future research to investigate the functions of these induced PSnMAPKs.

\section{Methods}

\section{Mycorrhizal fungal inoculum and plant material}

In our study, the $R$. irregularis (BGC BJ09) obtained from the Beijing Academy of Agriculture and Forestry Sciences (Beijing, China) was propagated with Zea mays as its host for three months. At harvest time, roots and growth substrates were collected as the fungal inoculum, which contained the fungal spores (20 spores/g). Annual cuttings of $P$. simoniixP. nigra were obtained from a plant nursery in Lindian County, Daqing City, Hei Longjiang Province, China. After washing, the cuttings were cut into small pieces, each of which had only one bud and the same length. These pieces were inserted in the nursery pot, and their growth substrate was a mixture of soil, sand, and vermiculite $(\mathrm{V}: \mathrm{V}: \mathrm{V}=1: 1: 1)$. One month later, they germinated and were inserted into the same substrate to take roots. After rooting, seedlings were transferred into plots with $1 \mathrm{~kg}$ mixture of soil and sand $(\mathrm{V}: \mathrm{V}=1: 1)$.

\section{Experimental design}

The experiment was a completely random design with two water conditions (WW: well-watered, DS: drought stress) and two AMF treatments (AM: inoculated with $R$. irregularis, NM: not inoculated with $R$.

irregularis). Based on the previous experience, WW treatment was considered as $70 \% \sim 75 \%$ of maximum water capacity of the sterilized soil/sand mixture, and DS treatment was considered as $30 \% \sim 35 \%$ of the maximum water capacity of the sterilized soil/sand mixture. There were four treatments in the present experiment, each treatment had 24 pots, 96 pots in total.

\section{Growth substrate and Plant culture}

The growth substrate was composed of autoclaved soil and sand. Before being put into the pots, the soil was sieved $(2 \mathrm{~mm})$ and autoclaved $\left(121^{\circ} \mathrm{C}, 2 \mathrm{~h}\right)$ and the sand was washed and dried $\left(180^{\circ} \mathrm{C}\right)$. The sterilized soil/sand mixture contained available nitrogen, $37.68 \mathrm{mg} \mathrm{kg}^{-1}$; available phosphorous, $11.28 \mathrm{mg} \mathrm{kg}^{-1}$; and available potassium, $139.10 \mathrm{mg} \mathrm{kg}^{-1}$, and the $\mathrm{pH}$ value was 7.7. At the time of transferring the seedlings, $20 \mathrm{~g}$ mycorrhizal inoculums were applied to seedlings as the AM treatment. The NM treatment received $20 \mathrm{~g}$ autoclaved mycorrhizal inoculums. Before the drought treatment, keeping the sterilized soil/sand mixture 
moisture content at $70 \% \sim 75 \%$ of maximum water capacity by weighing every day. After one week, half of the mycorrhizal and non-mycorrhizal plants were exposed to the $30 \% \sim 35 \%$ of maximum water capacity for 12 weeks, and the other half was continued to grow under well-watered conditions for 12 weeks. Seedlings were placed in the greenhouse, where the temperature was $25 \sim 28^{\circ} \mathrm{C}$, and the light intensity was $3000 \mathrm{Ix}$. During the experiment, $50 \mathrm{~mL}$ of Hoagland solution with $1 / 10$ of the phosphate was weekly added into each pot for normal growth of seedlings.

\section{Determination of mycorrhizal colonization}

Roots at the same position on seedlings from four treatments were cut. After washing with tap water, the roots were decolorized with $5 \% \mathrm{KOH}$ solution in a water bath at $90^{\circ} \mathrm{C}$ for $20 \mathrm{~min}$. After cooling, the roots were acidified with $2 \% \mathrm{HCl}$ solution, and they were dyed with $1 \%$ Trypan at $90{ }^{\circ} \mathrm{C}$ for $15 \mathrm{~min}$. Finally, the roots were decolorized with a mixture of lactic acid and glycerin $(\mathrm{V}: \mathrm{V}=1: 1)$ at room temperature. A compound microscope (Olympus BX43, Japan) was used to detect the AM colonization rate of each sample using the cross method [22].

\section{Determination of Photosynthesis}

Before harvest, the photosynthesis parameters of seedlings were determined using a Li-6400 Portable Photosynthesis System (Li-Cor Inc, Lincoln, NE, United States) on a sunny day from 9:00 to 11:00 am. Photosynthetic rate $(\mathrm{Pn})$, stomatal conductance $(\mathrm{gs})$, intercellular $\mathrm{CO}_{2}$ concentration $(\mathrm{Ci})$, and transpiration rate $(\mathrm{Tr})$ were measured after steady-state of gas exchange. Leaves on the same position were selected and leaves of each seedling used for measurement should be fully expanded, three leaves were selected for each seedling.

\section{Determination of active substances}

For MDA, $0.1 \mathrm{~g}$ frozen leaves and roots samples ground in liquid nitrogen were exacted with a solution containing $0.3 \%$ 2-thiobarbituric acid (TBA) and 10\% trichloroacetic acid (TCA). The MDA concentration was determined using the TBA reaction method, according to the method described by Hodges et al. [23]. For $\mathrm{H}_{2} \mathrm{O}_{2}$, $0.1 \mathrm{~g}$ of frozen leaves and roots samples were ground with $1 \mathrm{~mL}$ of precooled acetone solution, and $\mathrm{H}_{2} \mathrm{O}_{2}$ concentration was determined by the method described by Patterson et al. [24]. For proline, $0.1 \mathrm{~g}$ frozen leaves and roots samples ground in liquid nitrogen were exacted with $3 \%$ sulfosalicylic acid solution. The proline concentration was determined according to the method described by Bates et al. [25]. For soluble sugar, $0.1 \mathrm{~g}$ frozen leaves and roots samples were ground with $1 \mathrm{~mL} 75 \%$ methanol and determined following the method described by Shi et al. [26].

\section{Determination of antioxidant enzyme activity}

$0.1 \mathrm{~g}$ frozen leaves and roots samples ground in liquid nitrogen were exacted with $1 \mathrm{~mL} 50 \mathrm{mM}$ potassium phosphate buffer containing $1 \%$ polyvinylpyrrolidone (PVP). The homogenates were centrifuged at $12000 \times g$ for $20 \mathrm{~min}$ at $4{ }^{\circ} \mathrm{C}$ and the supernatants were used for the enzyme assays. The activity of SOD was measured by monitoring the inhibition of photochemical reduction of nitroblue tetrazole (NBT) [27]. CAT activity was determined by the method of Maehly and Chance [28]. 


\section{MAPK genes fragment cloning and quantitative expression}

After three months, the leaves and roots samples were ground completely in liquid nitrogen. Total RNA was extracted using a TIANGEN ${ }^{\circledR}$ RNAprep pure Plant Kit (Tiangen, Beijing, China) following the manufacturer's protocol and then $1 \mathrm{mg}$ total RNA was used for reverse transcription following the manufacturer's protocol of HiScript ${ }^{\circledR}$ II Q RT SuperMix for qPCR (+gDNA wiper) (Vazyme, Nanjing, China). The sequences of MAPK family genes of $P$. simonii $\times$ P. nigra were obtained using the MAPKs of $P$. trichocarpa to perform a homology search in Blast of NCBI based on the transcriptome data of $P$. simonii $\times$ P. nigra (Database Number: SRP267437). The accession numbers of MAPKs of $P$. trichocarpa in NCBI used for blast were listed in Table 3. Primers used for cloning were designed using Primer Tool of NCBI were listed in Table 4. Phylogenetic tree was constructed with MAPKs of Arabidopsis thaliana, Populus trichocarpa, rice, cotton, and maize. Muscle was used for multisequence comparison, and maximum likelihood method was used for tree construction in Mega-X software.

For the qRT-PCR assay, the reaction system is $10 \mu \mathrm{L}$, which included $5 \mu \mathrm{L}$ ChamQ SYBR ${ }^{\circledR}$ qPCR Master Mix (Vazyme, Nanjing, China), $1 \mu \mathrm{L}$ diluted cDNA template, $0.5 \mu \mathrm{L}$ forward and reverse primers and $3 \mu \mathrm{L}$ sterilized $\mathrm{ddH}_{2} \mathrm{O}$. Primers used in our experiment for qRT-PCR were listed in Table 5, which were designed by Primer Tool of NCBI, and the specificity were checked using the Blast Tool of NCBI. The qRT-PCR amplification program was as follows: $95^{\circ} \mathrm{C}$ for $30 \mathrm{~s}, 40$ cycles at $95^{\circ} \mathrm{C}$ for $10 \mathrm{~s}$, annealing at the annealing temperature for $10 \mathrm{~s}$ and extending at $72{ }^{\circ} \mathrm{C}$ for $20 \mathrm{~s}$, followed by heating from $65^{\circ} \mathrm{C}$ to $95^{\circ} \mathrm{C}$ at a rate of $0.5^{\circ} \mathrm{C}$ every $5 \mathrm{~s}$. Four biological replicates were used for all genetic analyses, CYP (coding peptidyl-prolyl cis-trans isomerase 1 ) and EF1a (coding elongation factor 1 -alpha-like) were used as the endogenous control to calculate $\triangle$ Ct values. The dissociation curves were used to detected the amplification specificity of primers and the $2^{-\Delta \triangle \mathrm{Ct}}$ method was used to calculated the relative quantification value of genes. Heatmaps of MAPKs expressions in leaves and roots of $P$. simoniixP. nigra were drawn using ImageGP online website, and the data were standardized.

Table 1. The accession number of MAPKs of $P$. trichocarpa homologous to $P$. simonii $\times P$. nigra 


\begin{tabular}{|ll|}
\hline Gene & Accession Number \\
\hline MAPK3-1 & LOC7475044 \\
\hline MAPK3-2 & LOC7480100 \\
\hline MAPK7-1 & LOC18099245 \\
\hline MAPK7-2 & LOC7480142 \\
\hline MAPK9 & LOC7462485 \\
\hline MAPK12 & LOC7482883 \\
\hline MAPK15-1 & LOC7475929 \\
\hline MAPK15-2 & LOC7454239 \\
\hline MAPK17 & LOC7459782 \\
\hline MAPK19-1 & LOC18103326 \\
\hline MAPK19-2 & LOC7491363 \\
\hline MAPK2O-1 & LOC7464636 \\
\hline MAPK20-2 & LOC7480727 \\
\hline
\end{tabular}

Table 2. Primers for MAPKs cloning of $P$. simoniixP. nigra.

\begin{tabular}{|lll|}
\hline Gene name & Primer-forward $\left(5^{\prime} \sim 3^{\prime}\right)$ & Primer-reverse $\left(5^{\prime} \sim 3^{\prime}\right)$ \\
\hline MAPK3-1 & AACCATGGATAAGAGGCGGC & TGCGAGTCAAGTTGCTGGAT \\
\hline MAPK3-2 & CAGACGATCGGAGATGGCAA & TAACGACCGATGAGGATGCG \\
\hline MAPK7-1 & TCATTTTGGCTCCCGAGGAC & TAAGCCGTCAACTGATCGCA \\
\hline MAPK7-2 & CTTGCAATTTGGCTGCCTGT & AGTGGTTGCGACGACTTGAT \\
\hline MAPK12 & AGTGCCCAGCAAATCAGTCA & AGCGAGGAATGCTGGAAGTT \\
\hline MAPK15-1 & GAGCTCCGGAATTGTGTGGA & CAGTCAACACTTTGAGCCGC \\
\hline MAPK15-2 & CATGCTCTGGGCAATGATGC & GGCAAAGGGAGCTATGGTGT \\
\hline MAPK17 & ACGGGAGAGAGGGTTGCTAT & GCATGGCTACAGCATTTCGG \\
\hline MAPK19-1 & CTGAGTACGGGGATGCCAAT & GCAGTGTGGAGAGCTGGTTT \\
\hline MAPK19-2 & GACACGGGAACACCATCAGT & AGCCTTTGCAGTAGCCTGAG \\
\hline MAPK20-1 & TAGATGGGGCAAATGCGGTT & GCATCCTGCCCTCCTCTTTT \\
\hline MAPK20-2 & TCGTCCAACTGCTGAAGAGG & GTTGAACCAGCGCTGCAAAT \\
\hline
\end{tabular}


Table 3. Primers of $P$. simoniixP. nigra used in qRT-PCR.

\begin{tabular}{|lll|}
\hline Gene name & Primer-forward $\left(5^{\prime} \sim 3^{\prime}\right)$ & Primer-reverse $\left(5^{\prime} \sim 3^{\prime}\right)$ \\
\hline MAPK3-1 & AATTCGCCATGTCCTCGGTT & GCATTTCTCCACCGCTTTCG \\
\hline MAPK3-2 & CGGACAAACCCAAGATCAGA & CTCAGACTATACTGCTGCGAT \\
\hline MAPK7-1 & TTCTTAGACATATCCGGCAT & TAATTCAGCCCACGTAGCAA \\
\hline MAPK7-2 & AAGTATGTTCCGATTAAGCC & TCACATTCTCATGCCGGAT \\
\hline MAPK9 & TCTCAGAATACGTGTGGCATC & GCAGATTTCTTCACCGAGT \\
\hline MAPK12 & CATATTTTGACGGACTAGCAG & GTTCCTTAAAGCGATCCAC \\
\hline MAPK15-1 & TGCTTTTCTCTGTGGCGACT & GAAGAGCCTGAGCTGGTTGA \\
\hline MAPK15-2 & ATCCATATTTTAAGGGTCTGGC & TACATAAACCCAGTTGGCTCT \\
\hline MAPK17 & GTCTTTAGGATCAAATGCCAG & GCTGCTGATACAATTGCAC \\
\hline MAPK19-1 & CTTTATCAGATGTTACGAGCAC & TCGTAGCAACATAATCCGTCCA \\
\hline MAPK19-2 & CATAGGGTACAACCGATCCCA & TGTCTCACTTCCACGGTCCA \\
\hline MAPK20-1 & CATGTTCTTCCTTGCTCCC & TTGTTACCGCAAATCTAGCAC \\
\hline MAPK20-2 & ACTCAGAAGATCAACGTGTC & GGTCTCAAGAATACAGTGCAA \\
\hline CYP & GGCTAATTTTGCCGATGAGA & ACGTCCATCCCTTCAACAAC \\
\hline EFa & ATTGACAGGCGGTCTGGTAAGGAA & AAACGACCAAGTGGAGGATACGCT \\
\hline
\end{tabular}

\section{Statistical analysis}

All data were analyzed statistically by using the software SPSS 16.0 (SPSS 16.0 for windows, USA). Independent-sample T-test and one-way analysis of variance (ANOVA) $(P<0.05)$ were used to compare the differences among treatments.

\section{Declarations}

\section{Authors' Contributions}

Jing Tao: performed the experiments, analysed the data, and wrote the manuscript; Fengxin Dong and Yihan Wang: designed the experiment and provided experimental methods; Hui Chen: analysed the data; Ming Tang: provided financial support and revised the manuscript.

\section{Acknowledgments}

We thank the administrator of nursery in Lindian County, Daqing City, Heilongjiang Province for providing poplar cuttings. 


\section{Funding}

This research was funded by the National Natural Science Foundation of China (32071639), the National Key Research and Development Program of China (2018YFD0600203-3).

\section{Availability of data and materials}

All data generated or analysed during this study are included in this manuscript, and the datasets used and/or analysed during the current study are available from the corresponding author on reasonable request.

\section{Ethics approval and consent to participate}

Not applicable.

\section{Consent for publication}

Not applicable.

\section{Competing interests}

The authors declare that they have no competing interests.

\section{References}

1. Santander C, Aroca R, Manuel Ruiz-Lozano J, Olave J, Cartes P, Borie F, et al. Arbuscular mycorrhiza effects on plant performance under osmotic stress. Mycorrhiza. 2017, 27:639-657.

2. Joshi R, Wani SH, Singh B, Bohra A, Dar ZA, Lone AA, et al. Transcription Factors and Plants Response to Drought Stress: Current Understanding and Future Directions. Front Plant Sci. 2016, 7:1029.

3. Anjum SA, Xie XY, Wang LC, Saleem MF, Man C, Lei W. Morphological, physiological and biochemical responses of plants to drought stress. Afr J Biomed Res. 2011, 6:2026-2032.

4. Choudhury FK, Rivero RM, Blumwald E, Mittler R. Reactive oxygen species, abiotic stress and stress combination. Plant J. 2017, 90:856-867.

5. Zhang T, Hu Y, Zhang K, Tian C, Guo J. Arbuscular mycorrhizal fungi improve plant growth of Ricinus communis by altering photosynthetic properties and increasing pigments under drought and salt stress. Ind Crops Prod. 2018, 117:13-19.

6. Latef AAHA, He C. Effect of arbuscular mycorrhizal fungi on growth, mineral nutrition, antioxidant enzymes activity and fruit yield of tomato grown under salinity stress. Sci. Hortic. 2011, 127:228-233.

7. Caverzan A, Passaia G, Rosa SB, Ribeiro CW, Lazzarotto F, Margis-Pinheiro M. Plant responses to stresses: Role of ascorbate peroxidase in the antioxidant protection. Mol. Genet. Genom. 2012, 35:1011-1019.

8. Wu F, Fang F, Wu N, Li L, Tang M. Nitrate Transporter Gene Expression and Kinetics of Nitrate Uptake by Populus $\times$ canadensis 'Neva' in Relation to Arbuscular Mycorrhizal Fungi and Nitrogen Availability. Front. Microbiol. 2020, 11.

9. Cely MVT, de Oliveira AG, de Freitas VF, de Luca MB, Barazetti AR, dos Santos IMO, et al. Inoculant of Arbuscular Mycorrhizal Fungi (Rhizophagus clarus) Increase Yield of Soybean and Cotton under Field 
Conditions. Front. Microbiol. 2016, 7.

10. Asmelash F, Bekele T, Birhane E. The Potential Role of Arbuscular Mycorrhizal Fungi in the Restoration of Degraded Lands. Front. Microbiol.. 2016, 7.

11. Chen M, Arato M, Borghi L, Nouri E, Reinhardt D. Beneficial Services of Arbuscular Mycorrhizal Fungi From Ecology to Application. Front Plant Sci. 2018, 9.

12. Wu F, Zhang H, Fang F, Wu N, Zhang Y, Tang M. Effects of Nitrogen and Exogenous Rhizophagus irregularis on the Nutrient Status, Photosynthesis and Leaf Anatomy of Populus $x$ canadensis 'Neva'. J Plant Growth Regul.2017, 36:824-835.

13. Danquah A, de Zelicourt A, Boudsocq M, Neubauer J, Frey NFD, Leonhardt N, et al. Identification and characterization of an ABA-activated MAP kinase cascade in Arabidopsis thaliana. Plant J. 2015, 82:232244.

14. Xiong $L$, Yang Y. Disease resistance and abiotic stress tolerance in rice are inversely modulated by an abscisic acid-inducible mitogen-activated protein kinase. Plant Cell. 2003, 15:745-759.

15. Rohila J, Yang Y. Rice Mitogen-activated Protein Kinase Gene Family and Its Role in Biotic and Abiotic Stress Response. J Integr Plant Biol. 2007, 49:751-759.

16. Zhang L, Xi D, Li S, Gao Z, Zhao S, Shi J, et al. A cotton group C MAP kinase gene, GhMPK2, positively regulates salt and drought tolerance in tobacco. Plant Mol Biol. 2011, 77:17-31.

17. Ning J, Li X, Hicks LM, Xiong L. A Raf-Like MAPKKK Gene DSM1 Mediates Drought Resistance through Reactive Oxygen Species Scavenging in Rice. Plant Physiol. 2010, 152:876-890.

18. Long L, Gao W, Xu L, Liu M, Luo X, He X, et al. GbMPK3, a mitogen-activated protein kinase from cotton, enhances drought and oxidative stress tolerance in tobacco. Plant Cell, Tissue Organ Cult. 2014, 116:153162.

19. Xu Q. Spatiotemporal Variation of Water Use Efficiency and Its Influencing Factors in Arid and Semi-Arid Areas of China. Geogr. Res. 2021, 10:126-136.

20. Li Z, Wu N, Liu T, Chen H, Tang M. Effect of arbuscular mycorrhizal inoculation on water status and photosynthesis of Populus cathayana males and females under water stress. Physiol Plant. 2015, 155:192-204.

21. Li Z, Wu N, Liu T, Tang M, Chen H. Gender-related responses of dioecious plant Populus cathayana to AMF, drought and planting pattern. Sci Rep. 2020, 10:11530.

22. Finlay RD, Lindahl BD, Taylor AFS. Chapter 13 Responses of mycorrhizal fungi to stress. In: British Mycological Society Symposia Series. vol. 27: Academic Press; 2008. p.: 201-219.

23. Bahadur A, Batool A, Nasir F, Jiang S, Qin M, Zhang Q, et al. Mechanistic Insights into Arbuscular Mycorrhizal Fungi-Mediated Drought Stress Tolerance in Plants. Int J Mol Sci. 2019, 20:4199.

24. Liu F, Stützel H. Biomass partitioning, specific leaf area, and water use efficiency of vegetable amaranth (Amaranthus spp.) in response to drought stress. Sci Hortic. 2004, 102:15-27.

25. Hu W, Zhang H, Chen H, Tang M. Arbuscular mycorrhizas influence Lycium barbarum tolerance of water stress in a hot environment. Mycorrhiza. 2017, 27:451-463.

26. Hura T, Hura K, Grzesiak M, Rzepka A. Effect of long-term drought stress on leaf gas exchange and fluorescence parameters in C-3 and C-4 plants. Acta Physiol Plant. 2007, 29:103-113. 
27. Yan W, Zhong Y, Shangguan Z. A meta-analysis of leaf gas exchange and water status responses to drought. Sci Rep. 2016, 6:20917.

28. Auge RM. Water relations, drought and vesicular-arbuscular mycorrhizal symbiosis. Mycorrhiza. 2001, 11:3-42.

29. Raghavendra AS, Gonugunta VK, Christmann A, Grill E. ABA perception and signalling. Trends Plant Sci. 2010, 15:395-401.

30. Chaves MM, Flexas J, Pinheiro C. Photosynthesis under drought and salt stress: regulation mechanisms from whole plant to cell. Ann Bot. 2009, 103:551-560.

31. Zhu XC, Song FB, Xu HW. Arbuscular mycorrhizae improves low temperature stress in maize via alterations in host water status and photosynthesis. Plant Soil. 2010, 331:129-137.

32. Chen S, Zhao H, Zou C, Li Y, Chen Y, Wang Z, et al. Combined Inoculation with Multiple Arbuscular Mycorrhizal Fungi Improves Growth, Nutrient Uptake and Photosynthesis in Cucumber Seedlings. Front Microbiol. 2017, 8:2516.

33. Amiri R, Nikbakht A, Etemadi N. Alleviation of drought stress on rose geranium Pelargonium graveolens (L.) Herit. in terms of antioxidant activity and secondary metabolites by mycorrhizal inoculation. Sci Hortic. 2015, 197:373-380.

34. Rani B, Madan S, Pooja, Sharma KD, Kumari N, Kumar A. Mitigating the effect of drought stress on yield in wheat (Triticum aestivum) using arbuscular mycorrhiza fungi (Glomus mosseae). J Agric Sci 2018, 88:95100.

35. Mo Y, Wang Y, Yang R, Zheng J, Liu C, Li H, et al. Regulation of Plant Growth, Photosynthesis, Antioxidation and Osmosis by an Arbuscular Mycorrhizal Fungus in Watermelon Seedlings under WellWatered and Drought Conditions. Front Plant Sci. 2016, 7:644.

36. Zhang Y, Yao Q, Li J, Wang Y, Liu X, Hu Y, et al. Contributions of an arbuscular mycorrhizal fungus to growth and physiology of loquat (Eriobotrya japonica) plants subjected to drought stress. Mycol Prog. 2015, $14: 84$.

37. Tyagi J, Shrivastava N, Sharma AK, Varma A, Pudake RN. Mycorrhiza Fungus Rhizophagus intraradices Mediates Drought Tolerance in Eleusine coracana Seedlings. Preprints. 2018, 2018050064.

38. Manoharan PT, Shanmugaiah V, Balasubramanian N, Gomathinayagam S, Sharma MP, Muthuchelian K. Influence of AM fungi on the growth and physiological status of Erythrina variegata Linn. grown under different water stress conditions. Eur J Soil Biol. 2010, 46:151-156.

39. Doubkova P, Vlasakova E, Sudova R. Arbuscular mycorrhizal symbiosis alleviates drought stress imposed on Knautia arvensis plants in serpentine soil. Plant Soil. 2013, 370:149-161.

40. Abbaspour H, Saeidi-Sar S, Afshari H, Abdel-Wahhab MA. Tolerance of Mycorrhiza infected Pistachio (Pistacia vera L.) seedling to drought stress under glasshouse conditions. J. Plant Physiol. 2012, 169:704709.

41. Zou YN, Wu QS, Huang YM, Ni QD, He XH. Mycorrhizal-Mediated Lower Proline Accumulation in Poncirus trifoliata under Water Deficit Derives from the Integration of Inhibition of Proline Synthesis with Increase of Proline Degradation. Plos One. 2013, 8: e80568. 
42. Yooyongwech S, Samphumphuang T, Tisarum R, Theerawitaya C, Cha-um S. Arbuscular mycorrhizal fungi (AMF) improved water deficit tolerance in two different sweet potato genotypes involves osmotic adjustments via soluble sugar and free proline. Sci Hortic. 2016, 198:107-117.

43. Wu QS, Zou YN, He XH. Contributions of arbuscular mycorrhizal fungi to growth, photosynthesis, root morphology and ionic balance of citrus seedlings under salt stress. Acta Physiol Plant. 2010, 32:297-304.

44. Zou YN, Huang YM, Wu QS, He XH. Mycorrhiza-induced lower oxidative burst is related with higher antioxidant enzyme activities, net $\mathrm{H}_{2} \mathrm{O}_{2}$ effluxes, and $\mathrm{Ca}^{2+}$ influxes in trifoliate orange roots under drought stress. Mycorrhiza. 2015, 25:143-152.

45. Gong M, You X, Zhang Q. Effects of Glomus intraradices on the growth and reactive oxygen metabolism of foxtail millet under drought. Ann. Microbiol. 2015, 65:595-602.

46. Rani B, Madan S, Sharma KD, ja P, Berwal MK, Kumar A. Effect of Mycorrhizal Colonization on Nitrogen and Phosphorous Metabolism in Wheat (Triticum aestivum L.) under Water Deficit Stress. Curr Microbiol. 2017, 6:916-929.

47. Wang J, Ding H, Zhang A, Ma F, Cao J, Jiang M. A Novel Mitogen-Activated Protein Kinase Gene in Maize (Zea mays), ZmMPK3, is Involved in Response to Diverse Environmental Cues. J Integr Plant Biol. 2010, 52:442-452.

48. Ergen NZ, Thimmapuram J, Bohnert HJ, Budak H. Transcriptome pathways unique to dehydration tolerant relatives of modern wheat. Funct Integr Genomics. 2009, 9:377-396.

49. Zhang L, Xi D, Li S, Gao Z, Zhao S, Shi J, et al. A cotton group C MAP kinase gene, GhMPK2, positively regulates salt and drought tolerance in tobacco. Plant Mol Biol. 2011, 77:17-31.

50. Liu Z, Li Y, Ma L, Wei H, Zhang J, He X, et al. Coordinated Regulation of Arbuscular Mycorrhizal Fungi and Soybean MAPK Pathway Genes Improved Mycorrhizal Soybean Drought Tolerance. Mol Plant Microbe Interact. 2015, 28:408-419.

51. Huang D, Ma M, Wang Q, Zhang M, Jing G, Li C, et al. Arbuscular mycorrhizal fungi enhanced drought resistance in apple by regulating genes in the MAPK pathway. Plant Physiol Biochem. 2020, 149:245-255.

52. Rentel M, Lecourieux D, Lecourieux Ouaked F, Usher S, Petersen L, Okamoto H, et al. OXI1 kinase is necessary for oxidative burst-mediated signaling in Arabidopsis. Nature. 2004, 427:858-861.

53. Zhang A, Jiang M, Zhang J, Tan M, Hu X. Mitogen-Activated Protein Kinase Is Involved in Abscisic AcidInduced Antioxidant Defense and Acts Downstream of Reactive Oxygen Species Production in Leaves of Maize Plants. Plant Physiol. 2006, 141:475-487.

54. Im JH, Lee H, Kim J, Kim HB, An CS. Soybean MAPK, GMK1 is dually regulated by phosphatidic acid and hydrogen peroxide and translocated to nucleus during salt stress. Mol Cells. 2012, 34:271-278.

\section{Figures}




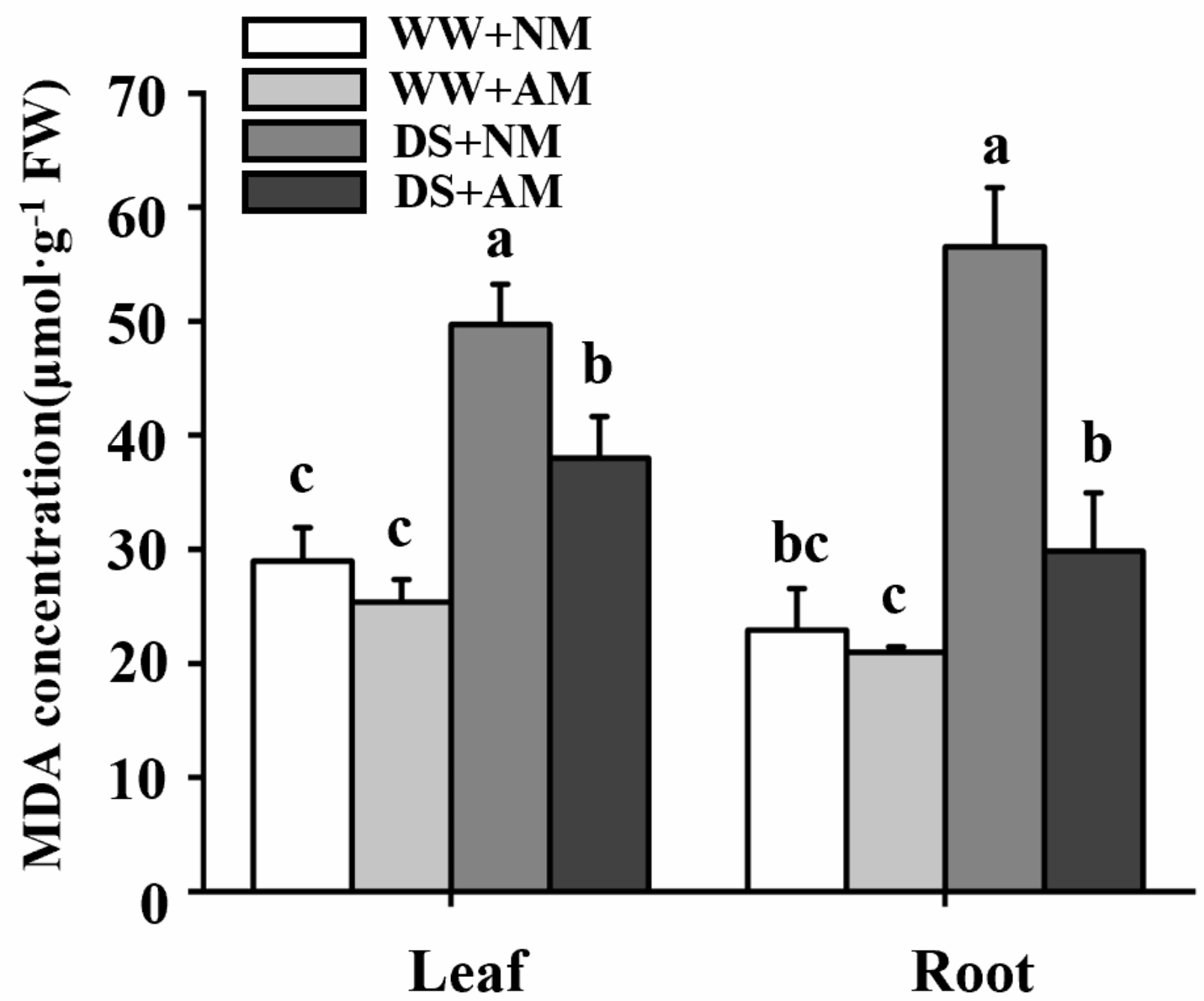

Figure 1

Effects of R. intraradices on leaf and root MDA concentration of P. simoniixP. nigra seedlings subjected to wellwatered (WW) and drought stress (DS). NM: nonmycorrhizal seedling; AM: mycorrhizal seedlings. Data (means $\pm S D, n=4)$ followed by different letters above the bars indicate significant differences $(P<0.05)$ between treatments. 


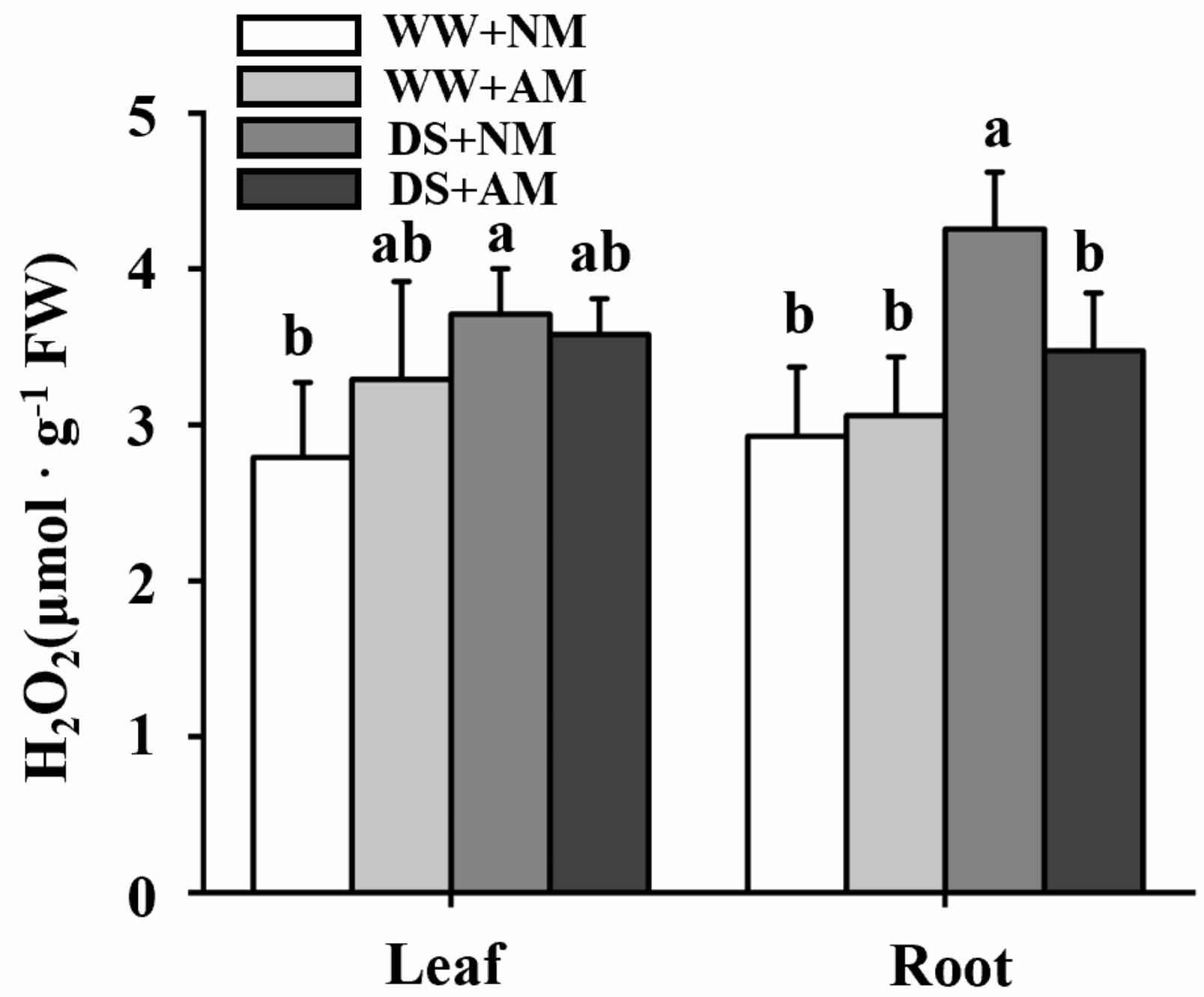

Figure 2

Effects of R. intraradices on leaf and root $\mathrm{H} 2 \mathrm{O} 2$ concentration of P. simoniixP. nigra seedlings subjected to well-watered (WW) and drought stress (DS). NM: nonmycorrhizal seedling; AM: mycorrhizal seedlings. Data (means $\pm S D, n=4)$ followed by different letters above the bars indicate significant differences $(P<0.05)$ between treatments. 


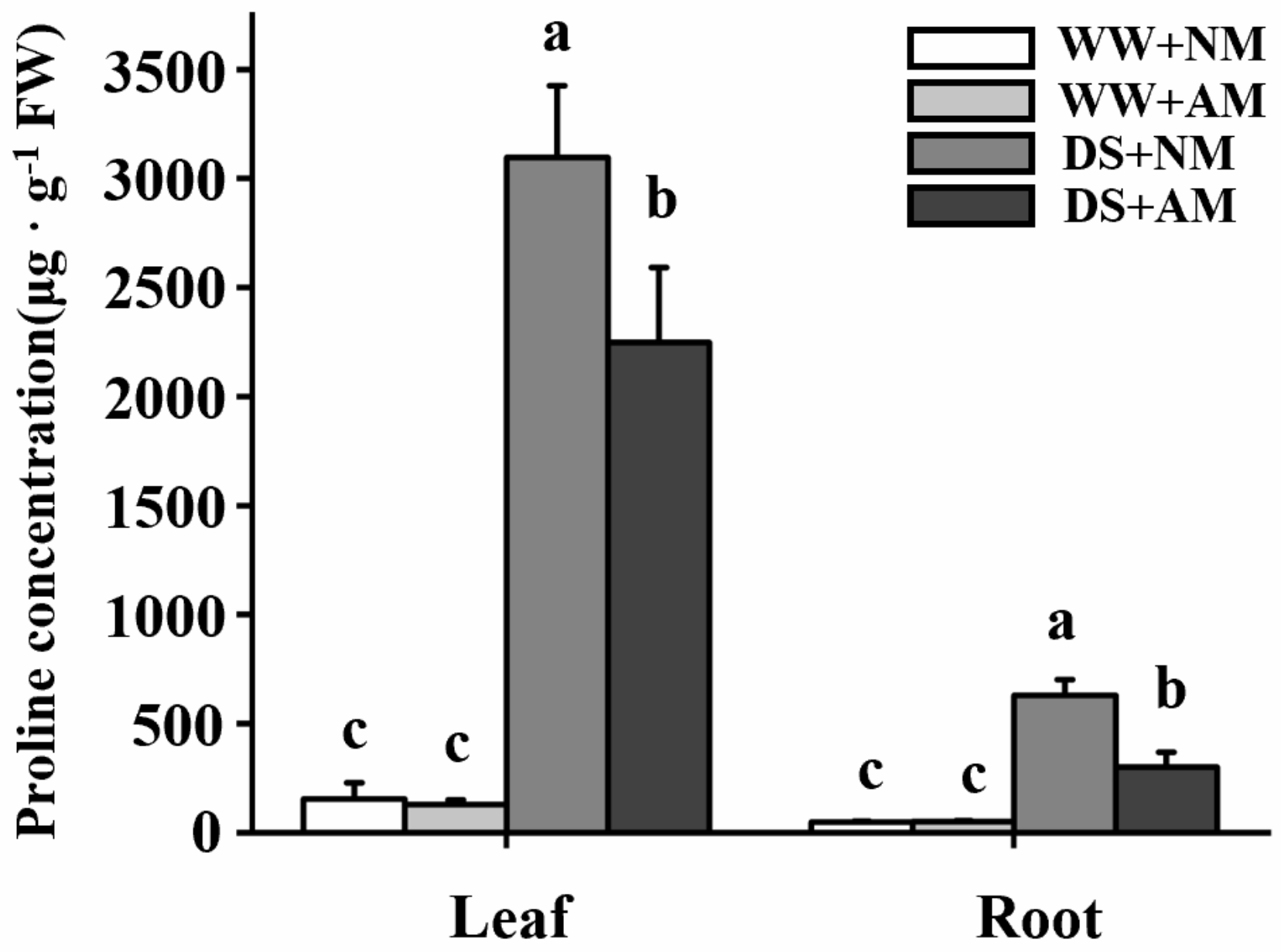

Figure 3

Effects of R. intraradices on leaf and root proline concentration of P. simoniixP. nigra seedlings subjected to well-watered (WW) and drought stress (DS). NM: nonmycorrhizal seedling; AM: mycorrhizal seedlings. Data (means $\pm S D, n=4)$ followed by different letters above the bars indicate significant differences $(P<0.05)$ between treatments. 


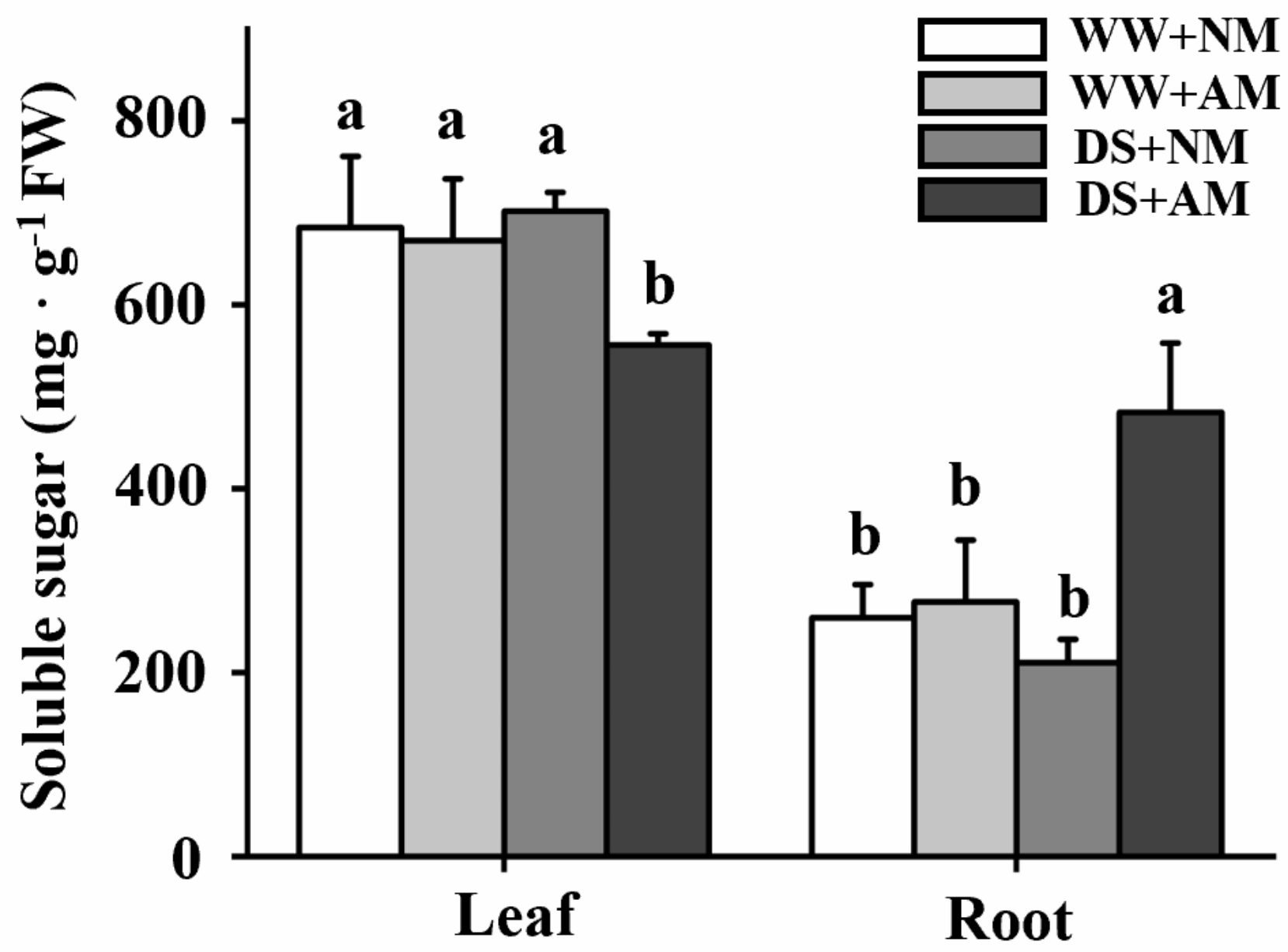

Figure 4

Effects of R. intraradices on leaf and root soluble sugar concentration of P. simoniixP. nigra seedlings subjected to well-watered (WW) and drought stress (DS). NM: nonmycorrhizal seedling; AM: mycorrhizal seedlings. Data (means $\pm S D, n=4$ ) followed by different letters above the bars indicate significant differences $(P<0.05)$ between treatments. 

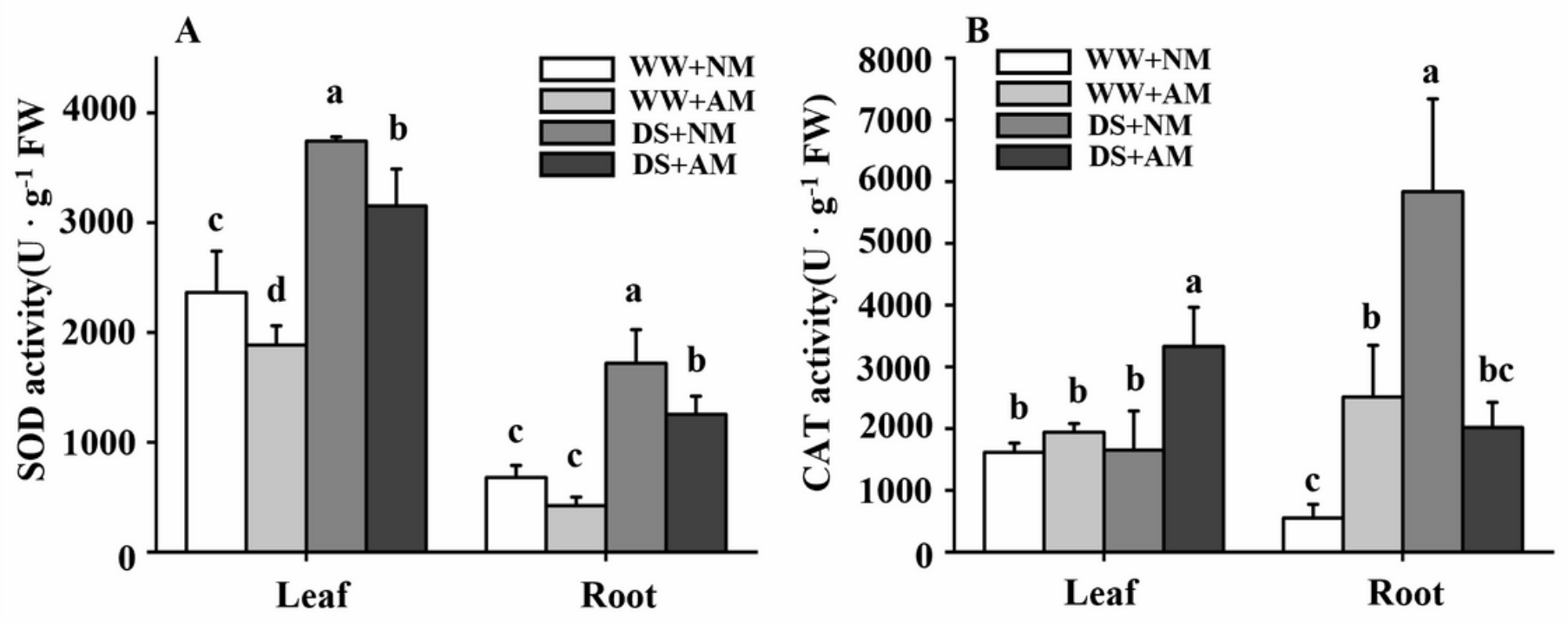

\section{Figure 5}

Effects of R. intraradices on leaf and root SOD and CAT activity of P. simoniixP. nigra seedlings subjected to well-watered (WW) and drought stress (DS). A: SOD activity; B: CAT activity; NM: nonmycorrhizal seedling; AM: mycorrhizal seedlings. Data (means $\pm S D, n=4$ ) followed by different letters above the bars indicate significant differences $(P<0.05)$ between treatments. 
Groups

A
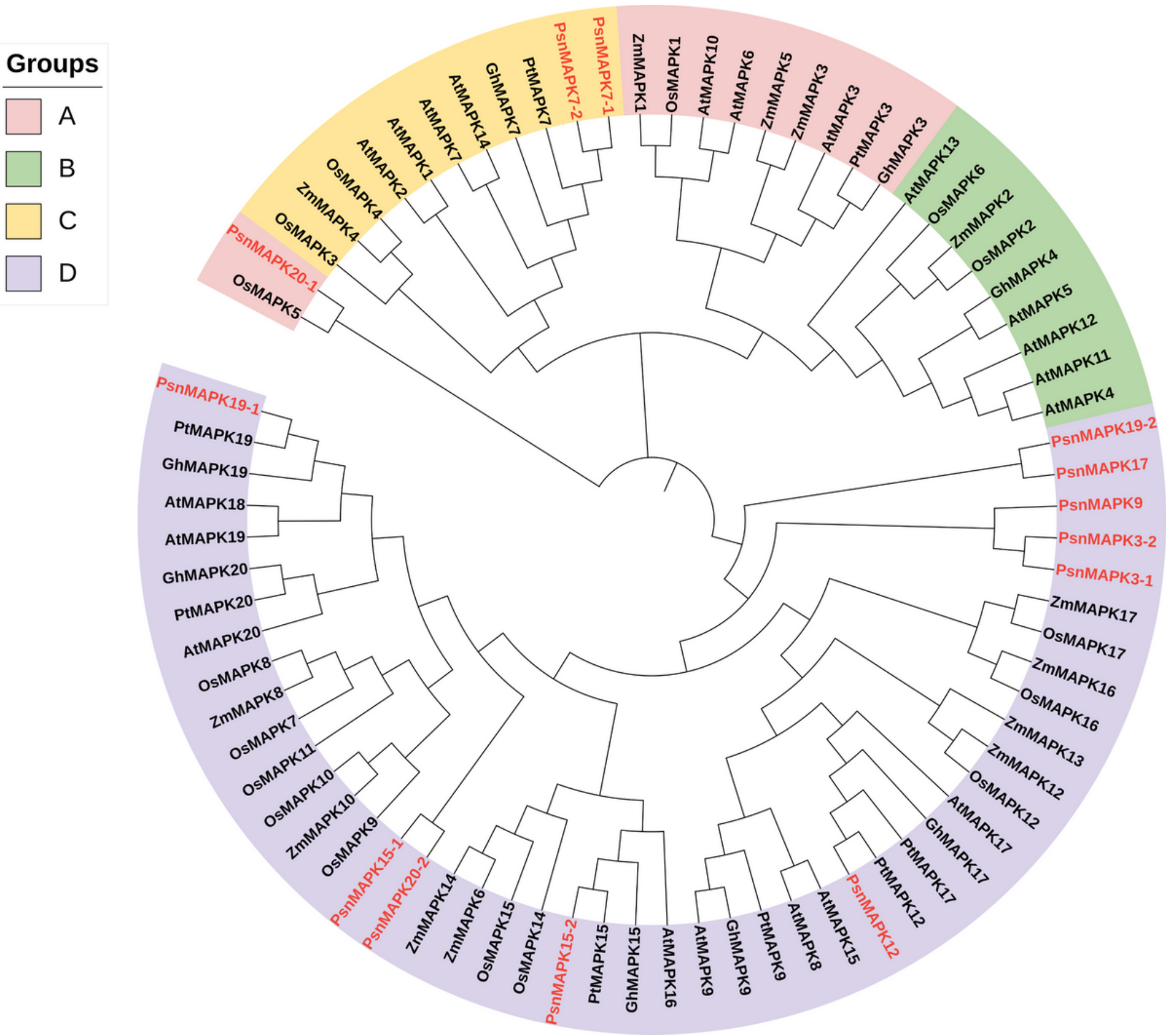

Figure 6

Phylogenetic tree of MAPKs of P P. simoniixP. nigra. Phylogenetic tree was constructed with MAPKs of Arabidopsis thaliana, Populus trichocarpa, rice, cotton, and maize. Muscle was used for multi-sequence comparison, and maximum likelihood method was used for tree construction in Mega-X software. 
A

Leaf

\begin{tabular}{|cccc|l|l|}
\hline a & a & b & b & PsnMAPK3-1 & 1 \\
a & a & b & b & PsnMAPK9 & 0.5 \\
b & a & c & bc & PsnMAPK19-1 & 0 \\
b & a & c & c & PsnMAPK20-2 & -0.5 \\
b & a & c & c & PsnMAPK12 & -1 \\
b & a & c & c & PsnMAPK17 \\
a & a & b & b & PsnMAPK15-1 \\
a & a & b & b & PsnMAPK15-2 \\
ab & a & b & ab & PsnMAPK3-2 \\
ab & a & c & bc & PsnMAPK19-2 \\
b & a & a & b & PsnMAPK7-1 \\
ns & ns & ns & ns & PsnMAPK7-2 \\
\hline b & c & a & bc & PsnMAPK20-1 \\
W+N & W+A & D+N & D+A &
\end{tabular}

\begin{tabular}{|c|c|c|c|c|c|}
\hline & \multicolumn{2}{|c|}{ B } & \multicolumn{3}{|c|}{ Root } \\
\hline b & a & b & b & PsnMAPK15-2 & \\
\hline $\mathbf{a b}$ & $\mathbf{a b}$ & a & b & PsnMAPK20-1 & $\begin{array}{l}1 \\
0.5\end{array}$ \\
\hline b & b & $\mathbf{a}$ & b & PsnMAPK3-1 & 0 \\
\hline b & b & $\mathbf{a}$ & b & PsnMAPK20-2 & -0.5 \\
\hline c & b & $\mathbf{a}$ & c & PsnMAPK7-2 & -1 \\
\hline b & b & $\mathbf{a}$ & b & PsnMAPK19-2 & \\
\hline bc & b & $\mathbf{a}$ & c & PsnMAPK15-1 & \\
\hline bc & b & a & c & PsnMAPK19-1 & \\
\hline c & $\mathbf{a}$ & $\mathbf{a}$ & b & PsnMAPK7-1 & \\
\hline b & $\mathbf{a}$ & $\mathbf{a}$ & $\mathbf{a b}$ & PsnMAPK3-2 & \\
\hline c & b & a & b & PsnMAPK12 & \\
\hline b & b & $\mathbf{a}$ & b & PsnMAPK9 & \\
\hline $\begin{array}{c}\mathbf{c} \\
\mathbf{W}+1\end{array}$ & $\begin{array}{c}\mathbf{b} \\
\mathbf{W}+\mathbf{A}\end{array}$ & a & b & PsnMAPK17 & \\
\hline
\end{tabular}

Figure 7

The heat maps of the expressions of PsnMAPKs in leaves and roots. A: Leaf; B: Root; WW: well-watered; DS: drought stress; NM: nonmycorrhizal seedling; AM: mycorrhizal seedlings. Data are standardized and different letters indicate significant differences $(P<0.05)$ between treatments. 